\title{
Clinical Outcomes of Patients Undergoing Kyphoplasty due to Vertebral Compression Fracture: A Retrospective Examination of 52 Patients
}

\author{
Ramazan Paşahan ${ }^{1}$, Emek Güldoğan ${ }^{2}$ \\ ${ }^{1}$ Department of Neurosurgery, Faculty of Medicine, Inonu University, Malatya, Turkey. \\ ${ }^{2}$ Department of Biostatistics and Medical Informatics, Faculty of Medicine, Inonu University, Malatya, Turkey \\ Received: 12 June 2020, Accepted: 21 August 2020, Published online: 31 August 2020 \\ (C) Ordu University Institute of Health Sciences, Turkey, 2020
}

\begin{abstract}
Objective: Osteoporosis, vertebral colon hemangiomata and metastatic tumors are among the causes of vertebral fractures. When treating vertebral fractures, the patient is rested, analgesic anti-inflammatory therapy and kyphoplasty are performed, and if there is an unstable fracture and/or neurological deficit, spinal cord decompression and stabilization are performed. Kyphoplasty is an effective method in stable fractures of the vertebrae ensuring minimal trauma, short surgical operation time and reduced pain in the early stages. In this study, clinical outcomes of 52 patients who underwent kyphoplasty at the Neurosurgery Clinic of Inonu University due to osteoporosis, vertebral hemangioma and spinal colon metastatic malignancies were discussed and presented along with the literature.

Method: In our study, quantitative data are presented with medians (minimums and maximums) or averages (standard deviations), and qualitative data are presented with counts (percentages). The assumption of normality was checked by using the Shapiro-Wilk test. Because the DEXA variable had a normal distribution $(p>0.05)$, one-way analysis of variance was utilized to analyze the difference between fracture types. The variables of age and Visual Analog Scale (VAS) were not normally distributed ( $p<0.05$ ), so the Kruskal Wallis $\mathrm{H}$ test was utilized to analyze the differences between fracture types. Pearson's chi-squared test was used to investigate how fracture sites were related to age groups and gender. $\mathrm{p}<.05$ was considered statistically significant.

Results: A total of 52 patients who did not require surgery at the Neurosurgery Clinic of Inonu University but underwent kyphoplasty between January 1, 2010 and April 1, 2020 were included in the study. Of these patients, 45 underwent kyphoplasty due to osteoporotic vertebral fractures, 3 due to vertebral hemangioma, and 4 due to spinal metastasis. All patients were compared in terms of age, gender, fracture sites, DEXA, preoperative VAS scores and VAS scores on day 20.

Conclusion: Kyphoplasty is an effective method for the treatment of stable vertebral fractures caused by osteoporosis, spinal metastases and vertebral hemangiomata
\end{abstract}

Key words: Kyphoplasty, Vertebral metastasis, Hemangioma.

Suggested Citation: Pasahan R, Guldogan E. Clinical Outcomes of Patients Undergoing Kyphoplasty due to Vertebral Compression Fracture: A Retrospective Examination of 52 Patients. Middle Black Sea Journal of Health Science, 2020; 6(2):183-189.

Address for correspondence/reprints:

Emek Güldoğan

Telephone number: + 90422341 0660/1313
ORCID-ID: 0000-0002-5436-8164

E-mail: emek.guldogan@inonu.edu.tr

DOI: $\quad 10.19127 / \mathrm{mbsjohs.750579}$ 


\section{Introduction}

Osteoporosis, trauma and spinal malignancies cause vertebral fractures. Osteoporosis causes a decrease in bone mineral density and an increase in a risk of fractures (Siris, Chen and Abbott, Kan et al. 2017). The incidence of osteoporotic vertebral fractures among people over 50 years of age is 307/100,000 (Hernlund et al. 2013). Vertebral colon is the most common site in bone metastases. Metastases can disrupt the integrity of vertebral corpus, make it susceptible to fracture under normal physiological stress, and cause neurological deficits (Uei and Tokuhashi 2018). Treatment of vertebral fractures aims to reduce pain, prevent new fractures and ensure spinal stabilization. In acute vertebral fractures, patients are rested, analgesic antiinflammatory therapy and kyphoplasty are performed, and if there is an unstable fracture and/or neurological deficit, spinal cord decompression and stabilization are performed. Kyphoplasty ensures minimal trauma, short surgical operation time and reduced pain in the early stages (Zuo et al. 2018). Vertebral hemangiomata make up 2-3\% of all spinal tumors. They are generally asymptomatic. In $1 \%$ of cases, they cause pain and inflict neurological deficits. Vertebroplasty/kyphoplasty, aggressive decompression stabilization, embolization and/or hybrid surgery can be performed in these patients (Nigro 2017).

In vertebral metastatic spinal tumors, patients complain of nagging pain and weight loss. Conservative treatments such as analgesics, chemotherapy, hormone therapy and radiotherapy may be effective for a short time. It is recommended to perform kyphoplasty in appropriate patients due to short life expectancy, high complications, long surgical time and long postoperative hospital stays in aggressive surgeries. It has been stated that kyphoplasty provides analgesia within 24-48 hours in $73 \%-92 \%$ of such patients. Kyphoplasty allows for biopsy during the procedure and causes little bleeding. It ensures that the height of the vertebral corpus is maintained and therefore provides sagittal and coronal balance and early pain management. Its cost is cheaper in the long term compared to the cost of other forms of treatment (Itagaki et al. 2012, Berenson et al. 2011).

In this study, clinical outcomes of 52 patients who underwent kyphoplasty at the Neurosurgery Clinic of Inonu University due to osteoporosis, vertebral hemangioma and spinal colon metastatic malignancies were discussed and presented along with the related literature.

\section{Methods}

\section{Patients}

Patients who underwent kyphoplasty at the Neurosurgery Clinic due to osteoporosis, vertebral hemangioma and spinal colon metastatic malignancies were enrolled in the present study. A total of 52 patients who did not require surgery at the Neurosurgery Clinic of Inonu University, Malatya, Turkey; but underwent kyphoplasty between January 1, 2010 and April 1, 2020 were included in the study. The related data of the patients were retrospectively gathered during the study period. About 2-4 cc cement was used during the kyphoplasty procedure. Instable vertebral fractures were not included in the study. Of these patients, 45 underwent kyphoplasty due to osteoporotic vertebral fractures, 3 due to vertebral hemangioma, and 4 due to spinal metastasis. The patients were followed up through radiography of scoliosis. The average duration of hospitalization was 48 hours in 52 patients after kyphoplasty.

\section{Data Analyses}

In the current study, quantitative data are presented with medians (minimums and maximums) or arithmetic means (standard deviations), and qualitative data are presented with counts (percentages). The assumption of normality was checked by using the Shapiro-Wilk test. Because the DEXA variable had a normal distribution $(p>0.05)$, one-way analysis of variance was utilized to analyze the difference between fracture types. The variables of age and Visual Analog Scale (VAS) were not normally distributed ( $p<0.05$ ), so, the Kruskal Wallis $\mathrm{H}$ test was utilized to analyze the differences between fracture types. Pearson's chi-squared test was used to investigate how fracture sites were related to age groups and gender. $\mathrm{p}<0.05$ was considered statistically significant. The IBM SPSS 26.0 Statistics program was used to conduct the analyses.

\section{Results}

The mean age of 45 patients with osteoporotic vertebral fractures was $64.04 \pm 13.20$ (ranging from 30 to 87$)$. Of the patients, $24(53.3 \%)$ were female, and $21(46.27 \%)$ were male. The number of patients under 65 years of age was $18(40.0 \%)$, and the number of patients aged 65 and older was $27(60.0 \%)$. Among the patients who were younger than 65 years of age, $7(38.9 \%)$ were women and $11(61.1 \%)$ were men, and of those who were 65 years old or older, 17 $(53.3 \%)$ were women and $10(46.7 \%)$ were men. The number of patients whose fracture site was T10 and above was $12(26.7 \%)$, the number of those whose 
fracture site was T11-L1 was 18 (40.0\%), and the number of those whose fracture site was L2 and below was $15(33.3 \%)$. There were statistically significant differences between the fracture sites in terms of age groups (Pearson's Chi-squared test, $\mathrm{p}=$ 0.01 , Table 1). The significant differences were between T10 and T11-L1 for each age category. There were no statistically significant differences between the fracture sites in terms of gender (Pearson's Chi-squared test, $\mathrm{p}=0.268$, Table 1 ).

There were no statistically significant differences between fracture sites in terms of the variables of VAS initial and VAS on day $20(p>0.05$, Table 2$)$. DEXA results of $41(91.11 \%)$ of these patients could be obtained, but the results of $4(8.89 \%)$ could not be obtained. The average value of patients undergoing DEXA was between $2.59 \pm 0.72$ (ranging from 1 to 4.5). There was no statistically significant difference between the fracture sites in terms of the DEXA variable (One-way analysis of variance, $p=0.291$, Table 2).

There was no statistically significant difference between the initial VAS scores and the VAS scores after 20 days in all patients who underwent kyphoplasty due to osteoporotic vertebral fractures (Wilcoxon Paired Two-Sample Test, $\mathrm{p}=0.0001$ ). Six $(13.33 \%)$ patients had fractures in two sites, and 39 $(86.67 \%)$ patients had fractures in one site.

In a one-year follow-up period, there were no features in $40(88.9 \%)$ of the patients, whereas there were vertebral fractures in other sites in $5(11.1 \%)$ of the patients. Of these patients, 4 were found to have fractures (T7, T12, L4, T7) at the first-year follow-up, and one (T7) at the 3-month follow-up at the neighboring segment. No patients had complications after kyphoplasty.

The average age of the patients undergoing kyphoplasty due to vertebral hemangioma was 46.33 for 3 patients, and all patients were female. The fracture sites were L1, L1 and T8, respectively. The average preoperative VAS score was 8 , and the follow-up VAS score on day 20 was 3. The DEXA average was -0.87 , and there was no osteoporosis. No patients developed complications after kyphoplasty (Table 3).

Of the patients who underwent kyphoplasty due to metastatic vertebral fractures, the average age of 4 patients one of whom had myeloma, 2 prostate cancer and 1 pulmonary cancer metastasis was 59.75 . One of them was a woman (25.0\%), and the other 3 were men (75.0\%). The fracture sites were the levels L4, L3, T7-8 and L3, respectively. The average of preoperative VAS scores was 7, and the follow-up VAS score average on day 20 was 3.5 . The patient with myeloma had a fracture in the neighboring vertebrae 3 months later. There were no complications during the operations (Table 3).

Table 1. Fracture sites in osteoporotic vertebral fractures in relation to age and gender

\begin{tabular}{|c|c|c|c|c|c|c|}
\hline \multirow[b]{2}{*}{ Variable } & \multirow[b]{2}{*}{$\begin{array}{l}\text { Variable } \\
\text { Classes }\end{array}$} & \multicolumn{3}{|c|}{ Fracture Sites } & \multirow[b]{2}{*}{ Total } & \multirow[b]{2}{*}{$\mathbf{p}^{*}$} \\
\hline & & $\begin{array}{c}\text { T10 and above } \\
\text { n }(\%)\end{array}$ & $\begin{array}{c}\text { T11-L1 } \\
\text { n }(\%)\end{array}$ & $\begin{array}{c}\text { L2 and below } \\
n(\%)\end{array}$ & & \\
\hline \multirow{2}{*}{ Age } & $<65$ & $2^{a}(16.7)$ & $12^{\mathrm{b}}(66.7)$ & $4^{\mathrm{a}, \mathrm{b}}(26.7)$ & $18(40.0)$ & \multirow{2}{*}{0.01} \\
\hline & $>=65$ & $10^{\mathrm{a}}(83.3)$ & $6^{\mathrm{b}}(33.3)$ & $11^{\mathrm{a}, \mathrm{b}}(73.3)$ & $27(60.0)$ & \\
\hline \multirow{2}{*}{ Gender } & Female & $8(66.7)$ & $7(38.9)$ & $9(60.0)$ & $24(53.3)$ & \multirow{2}{*}{0.268} \\
\hline & Male & $4(33.3)$ & $11(61.1)$ & $6(40.0)$ & $21(46.7)$ & \\
\hline
\end{tabular}

*: Pearson' Chi-squared test. Values in the same row and sub-table that do not have the same superscript differ significantly at $\mathrm{p}<0.05$ for a two-way test for column ratios (Pearson's Chi-squared test with Bonferroni correction).

Table 2. Table of fracture sites in osteoporotic vertebral fractures in relation to the variables of age, VAS initial and VAS on day 20

\begin{tabular}{ccccc}
\hline Variables & \multicolumn{3}{c}{ Fracture Sites } & p \\
\cline { 2 - 4 } & $\begin{array}{c}\text { T10 and above } \\
(\mathbf{n = 1 2})\end{array}$ & $\begin{array}{c}\text { T11-L1 } \\
(\mathbf{n = 1 8})\end{array}$ & $\begin{array}{c}\text { L2 and below } \\
(\mathbf{n = 1 5})\end{array}$ & $0.302^{*}$ \\
\hline $\begin{array}{c}\text { VAS Initial } \\
{[\text { Median (Min-Max)] }} \\
\text { VAS after 20 days } \\
{[\text { Median (Min-Max)] }} \\
\text { DEXA }\end{array}$ & $6(6-8)$ & $8(6-8)$ & $8(6-8)$ & $0.096^{*}$ \\
(Mean \pm Standard Deviation) & $2(2-4)$ & $2(2-4)$ & $4(2-4)$ & $0.291^{* *}$ \\
\hline
\end{tabular}

*: Kruskal Wallis $\mathrm{H}$ test, **: One-way analysis of variance. 
Table 3. Age, gender, fracture site, VAS scores and DEXA values of patients who had vertebral hemangioma and vertebral metastasis

\begin{tabular}{lcccccc}
\hline \multicolumn{1}{c}{ Variables } & Age & Gender & $\begin{array}{c}\text { Fracture } \\
\text { site }\end{array}$ & $\begin{array}{c}\text { Prior to } \\
\text { Operation } \\
\text { VAS }\end{array}$ & Day 20 VAS & DEXA \\
\hline Hemangioma & 65 & Female & L1 & 8 & 4 & 0.8 \\
Hemangioma & 38 & Female & L1 & 8 & 3 & 1.2 \\
Hemangioma & 36 & Female & T8 & 8 & 2 & 0.6 \\
Myeloma & 52 & Female & L4 & 6 & 4 & - \\
Prostate metastasis & 55 & Male & L3 & 8 & 3 & - \\
Prostate metastasis & 72 & Male & L3 & 6 & 4 & - \\
Lung metastasis & 60 & Male & T7-8 & 8 & 3 & - \\
\hline
\end{tabular}

\section{Discussion}

The incidence of vertebral fractures owing to osteoporosis is increasing on a continuous basis. They can lead to consequences with high mortality such as post-fracture pain, kyphosis or scoliosis, inactivityrelated stroke, depression, DVT, and pulmonary embolism (Zhang et al. 2017b). Of patients over the age of $50,30-50 \%$ are in the risk group for osteoporotic vertebral fractures (OVFs), and this risk increases over the age of 65 . In Europe, the incidence of OVF is 570/100,000 among men and 1070/100,000 among women, and in South Korea, the 5-year OVF incidence is $852 / 100,000$ (Choi et al. 2020). In a study of Peh et al. on 155 patients, $79.97 \%$ of people diagnosed with OVF were reported to be female, and the average age was 73.6 (Peh, Gilula and Peck 2002). Age and gender in our study parallel the relevant literature. Research has shown that vertebral fractures are usually seen in the T8-L3 range and most commonly occur in the 12th thoracic and 1st lumbar vertebrae (Peh et al. 2002, Bolat and Mistık 2019). In our study, vertebral fractures were common in the T12-L1 region in all patient groups, and this is consistent with the literature. Levels at which agedependent vertebral fractures occur linked to osteoporosis have not been reported in the literature. In our study, vertebral fractures were more frequently detected above T10 and below L2 in patients 65 years of age or older. What draws attention is that these patients were usually kyphotic patients, and this can be explained by the change in the center of gravity on the sagittal plane. However, more biomechanical studies are needed. The incidence of fracture development in the neighboring vertebrae after kyphoplasty increases (Koyuncu et al. 1996). Zhu K et al. (2013) and Movrin I et al. (2012) have reported in their retrospective studies that patients undergoing kyphoplasty have few fractures of the neighboring vertebrae. It has been reported that an intact vertebral corpus and the volume of cement applied are risk factors for cement leakage in patients undergoing kyphoplasty. In balloon kyphoplasty, the incidence of neighboring vertebral fractures increases due to the change of the Cobb's angle (Chen et al. 2020). The rise of incidence of neighboring vertebral fractures is still controversial. In our study, 5 patients were found to have vertebral fractures in another site at the follow-up after kyphoplasty, and none of our patients had complications due to cement leakage. If bone mineral density is above 2.5 based on DEXA, the risk of fractures increases, and density decreases depending on age but increases the risk of fracture with advancing age (Kanis et al. 2004, Cakir et al. 2009). In our study, the DEXA average was less than -2.5 consistent with the literature. There was no statistically significant difference when the DEXA was compared based on fracture levels. There has been a significant decrease in VAS scores after kyphoplasty application in all studies (Liu, Cao and Kong 2019). In our study, there was a significant decrease in patients undergoing kyphoplasty due to osteoporotic vertebral fractures. This shows the effectiveness of kyphoplasty.

In vertebral hemangiomata, $2 / 3$ of all hemangiomata are seen in the cranium and spinal colon. Vertebral hemangiomata constitute $2-3 \%$ of all spinal tumors, are usually benign, and $1 \%$ of them are symptomatic. Neurological symptoms accompany in $30-40 \%$ of symptomatic patients (Acosta Jr et al. 2006, SPINE, Chi, Manley and Chou 2005). The rate of bleeding, surgical duration and the rate of complications are high in vertebral hemangiomata during open surgery. Radiotherapy, vertebroplasty and/or kyphoplasty are an effective method in patients with only pain complaints. Decompression, embolization and aggressive surgery are recommended in patients with neurological deficits (Chi et al. 2005, Saracen and Kotwica 2018). VAS scores were used to determine the level of pain in a series of studies conducted on 110 patients. Complications developed in 3 patients (Saracen and Kotwica 2018). Our study parallels the literature. There were no complications in our patients during the operation. 
In metastatic vertebral fractures, tumors that metastasize to the spinal column are most commonly breast, lung, prostate and other tumors. Of metastatic vertebral tumors, $3 / 4$ form osteolytic tumors and $1 / 10$ form osteoblastic tumors (Georgy 2010, Zhang et al. 2017a). Of spinal colon metastases, $7 / 10$ involve the thoracic region, 2/10 involve the lumbar region and $1 / 10$ involve the cervical region, and bone involvement is observed in $3 / 5$ of newly diagnosed multiple myeloma patients (Gerszten and Welch 2000). Kyphoplasty and/or radiotherapy is an effective and alternative method because of short lifespan and for managing pain in metastatic spinal tumors. The risk of damage to the back wall is very high, particularly in metastatic osteolytic lesions when the balloon is inflated (Wang et al. 2016). Leakage of bone cement in up to $38 \%$ of cases was reported in some studies (Liu et al. 2017). Pflugmacher et al. (2006) found a significant decrease in VAS scores of 31 patients who underwent multiple myeloma kyphoplasty, after one year of follow-up. Early significant enhancement was observed in VAS scores in metastatic vertebral fractures (Wang et al. 2016, Liu et al. 2017, Zhou et al. 2019). There was a case of lung cancer metastasis in our study, and the cancer had metastasized to the thoracic region. This is consistent with the literature. Two patients with prostate cancer metastases and one patient with multiple myeloma are consistent with the literature, as well. No patients developed complications after kyphoplasty. However, a patient with multiple myeloma was diagnosed with fractures in the neighboring vertebrae three months later. It could not be understood whether the cause of this fractures in neighboring vertebrae was due to myeloma and/or kyphoplasty.

\section{Conclusion}

Medical diagnosis and care opportunities are growing in the world every passing day. As a result, the incidence of vertebral fractures due to osteoporosis, hemangioma and spinal metastases has been increasing. Due to long operative time, excessive bleeding, excess complications, long postoperative pain control for all patient groups and long hospital stay in aggressive vertebral surgeries, kyphoplasty is a suitable choice for stable vertebral fractures. Kyphoplasty is performed to control pain, prevent the development of deformity, improve the quality of life by preventing fatal complications due to immobilization, reduce costs and reduce the length of hospital stay. Kyphoplasty is an effective method for the treatment of stable vertebral fractures caused by osteoporosis, spinal metastases and vertebral hemangiomata.

Ethics Committee Approval: Ethics committee approval was received for this study from Scientific Research and Publication Ethics Committee of İnonu University. Ethics no: 2020/708.

Peer-review: Externally peer-reviewed.

Author Contributions: Concept- R.P., DesignE.G., Supervision- R.P., E.G., Literature ReviewR.P., E.G., Writing- R.P., E.G., Critical Review- R.P., E.G.

Conflict of Interest: No conflict of interest was declared by the authors.

Financial Disclosure: The authors declared that this study has not received no financial support.

\section{References}

Acosta Jr, F. L., C. F. Dowd, C. Chin, T. Tihan, C. P. Ames \& P. R. Weinstein. Current treatment strategies and outcomes in the management of symptomatic vertebral hemangiomas. Neurosurgery; 2006;58(2):287-295.

Berenson, J., R. Pflugmacher, P. Jarzem, J. Zonder, K. Schechtman, J. B. Tillman, L. Bastian, T. Ashraf, F. Vrionis \& C. P. F. E. Investigators. Balloon kyphoplasty versus non-surgical fracture management for treatment of painful vertebral body compression fractures in patients with cancer: a multicentre, randomised controlled trial. The lancet oncology; 2011;12(3):225-235.

Bolat, E. S. \& S. Mistık. Yerleşmiş Osteoporozda Teriparatid Tedavisinin Spinal Deformite İndeksi Üzerine Etkisi. Turk J Osteoporos; 2019;25:6-11.

Cakir, B., C. Carazzo, R. Schmidt, T. Mattes, H. Reichel \& W. Käfer. Adjacent segment mobility after rigid and semirigid instrumentation of the lumbar spine. Spine; 2009;34(12):1287-1291.

Chen, C., P. Fan, X. Xie \& Y. Wang. Risk Factors for Cement Leakage and Adjacent Vertebral Fractures in Kyphoplasty for Osteoporotic Vertebral Fractures. Clinical Spine Surgery; 2020;33(6):E251-E255.

Chi, J. H., G. T. Manley \& D. Chou. Pregnancyrelated vertebral hemangioma: case report, review of the literature, and management algorithm. Neurosurgical focus; 2005;19(3):1-7. 
Choi, S. H., D.-Y. Kim, J. W. Koo, S. G. Lee, S.-Y. Jeong \& C.-N. Kang. Incidence and management trends of osteoporotic vertebral compression fractures in South Korea: a nationwide populationbased study. Asian Spine Journal; 2020;14(2):220.

Georgy, B. A. Vertebroplasty technique in metastatic disease. Neuroimaging Clinics; 2010;20(2):169177.

Gerszten, P. C. \& W. C. Welch. Current surgical management of metastatic spinal disease. 2000.

Hernlund, E., A. Svedbom, M. Ivergård, J. Compston, C. Cooper, J. Stenmark, E. V. et. al. Osteoporosis in the European Union: medical management, epidemiology and economic burden. Archives of osteoporosis; 2013;8(1-2):136.

Itagaki, M. W., A. D. Talenfeld, S. W. Kwan, J. W. Brunner, K. E. Mortell \& M. C. Brunner. Percutaneous vertebroplasty and kyphoplasty for pathologic vertebral fractures in the Medicare population: safer and less expensive than open surgery. Journal of Vascular and Interventional Radiology; 2012;23(11):1423-1429.

Kan, S.-L., Z.-F. Yuan, L.-X. Chen, J.-C. Sun, G.-Z. Ning \& S.-Q. Feng. Which is best for osteoporotic vertebral compression fractures: balloon kyphoplasty, percutaneous vertebroplasty or nonsurgical treatment? A study protocol for a Bayesian network meta-analysis. BMJ open; 2017;7:e012937.

Kanis, J., O. Johnell, A. Odén, C. De Laet \& D. Mellstrom. Epidemiology of osteoporosis and fracture in men. Calcified tissue international; 2004;75(2):90-99.

Koyuncu, H., S. Karamehmetoğlu, C. Bahadır \& K. Akgün. Postmenopozal vertebral kompresyon fraktürü sıklığı: Yaş, boy, kilo, boy/kulaç, menopoz süresi ilişkisinin değerlendirilmesi. Osteoporoz Dünyasından; 1996;2:81-84.

Liu, Q., J. Cao \& J. Kong. Clinical effect of balloon kyphoplasty in elderly patients with multiple osteoporotic vertebral fracture. Nigerian journal of clinical practice; 2019;22(3):289.

Liu, Y., Y. Wang, L. Zhao, R. Song, H. Tan \& L. Wang. Effectiveness and safety of percutaneous vertebroplasty in the treatment of spinal metastatic tumor. Pakistan journal of medical sciences; 2017; 33(3):675.
Movrin, I. Adjacent level fracture after osteoporotic vertebral compression fracture: a nonrandomized prospective study comparing balloon kyphoplasty with conservative therapy. Wiener Klinische Wochenschrift;2012;124(9-10):304-311.

Nigro, L. Algorithm of treatment for extensive vertebral hemangiomas according to Tomita classification of vertebral tumors. Journal of Neurology and Neuroscience;2017;8(02).

Peh, W. C., L. A. Gilula \& D. D. Peck. Percutaneous vertebroplasty for severe osteoporotic vertebral body compression fractures. Radiology; 2002; 223(1):121-126.

Pflugmacher, R., F. Kandziora, R.-J. Schroeder, I. Melcher, N. Haas \& C. Klostermann. Percutaneous balloon kyphoplasty in the treatment of pathological vertebral body fracture and deformity in multiple myeloma: a one-year follow-up. Acta Radiologica; 2006;47(4):369376.

Saracen, A. \& Z. Kotwica. Vertebroplasty (PVP) is effective in the treatment of painful vertebral hemangiomas. Acta Orthopædica Belgica; 2018;84:105-107.

Siris, E., Y. Chen \& T. Abbott a, Barrett-Connor E, Miller PD, Wehren LE, Berger ML. Bone mineral density thresholds for pharmacological intervention to prevent fractures. Arch Intern Med.; 2004;164(10):1108-1112.

Uei, H. \& Y. Tokuhashi. Prognostic factors in patients with metastatic spine tumors derived from lung cancer-a novel scoring system for predicting life expectancy. World journal of surgical oncology;2018;16(1):131.

Wang, Y., H. Liu, B. Pi, H. Yang, Z. Qian \& X. Zhu. Clinical evaluation of percutaneous kyphoplasty in the treatment of osteolytic and osteoblastic metastatic vertebral lesions. International Journal of Surgery; 2016;30:161-165.

Zhang, H.-T., G.-D. Chen, H.-L. Yang \& Z.-P. Luo. Percutaneous kyphoplasty in the treatment of osteoblastic-related spinal metastases. Clinical spine surgery; 2017;30(2):80-84.

Zhang, Y.-L., L.-T. Shi, P.-F. Tang, Z.-J. Sun \& Y.H. Wang. Correlation analysis of osteoporotic vertebral compression fractures and spinal sagittal imbalance. Der Orthopäde; 2017;46(3):249-255. 
Zhou, Z., Y. Wang, Z. Sun \& Z. Qian. Safety of Cement Distribution Patterns in Metastatic Vertebral Tumors: A Retrospective Study. Medical science monitor: international medical journal of experimental and clinical research; 2019; 25:7228.

Zhu, K., Zhang C. Shao C. Research progress of secondary fracture of adjacent vertebral body after percutaneous vertebroplasty and percutaneous kyphoplasty. Zhongguo xiufu chongjian wai $\mathrm{Ke}$ Za Zhi 2013 Mar;27(3):369-73

Zuo, X.-H., X.-P. Zhu, H.-G. Bao, C.-J. Xu, H. Chen, X.-Z. Gao \& Q.-X. Zhang. Network meta-analysis of percutaneous vertebroplasty, percutaneous kyphoplasty, nerve block, and conservative treatment for nonsurgery options of acute/subacute and chronic osteoporotic vertebral compression fractures (OVCFs) in short-term and long-term effects. Medicine; 2018;97(29). 